\title{
Mechanism for domain wall pinning and potential landscape modification by artificially patterned traps in ferromagnetic nanowires
}

\author{
Dorothée Petit, Ana-Vanessa Jausovec, Huang T. Zeng, Emma Lewis, Liam O’Brien, Dan Read, and Russell P. Cowburn \\ Department of Physics, Nanoscale Magnetics Group, Blackett Laboratory, Imperial College London, \\ Prince Consort Road, London SW7 2BW, United Kingdom
}

(Received 28 October 2008; revised manuscript received 11 May 2009; published 8 June 2009)

\begin{abstract}
The interaction mechanism between transverse domain walls (TDWs) in Permalloy nanowires and artificially patterned traps is studied using high-sensitivity spatially resolved magneto-optical Kerr effect measurements and numerical simulations. T-shaped trap geometries are considered, where a DW traveling in the horizontal arm is pinned by the vertical arm. Pinning strengths as well as potential energy modifications created by the traps are measured, and the roles of the different DW characteristic parameters, such as the DW core orientation and the magnetic charge distribution within the DW, are presented. It is found that whether or not the core of the DW is aligned with the transverse arm of the T structure affects the shape of the main potential experienced by the DW, whereas the pinning strength strongly depends on which side of the V-shaped TDW interacts with the trap. The role of the magnetostatic interaction between the charge of the DW and the charge present at the junction is discussed.
\end{abstract}

DOI: 10.1103/PhysRevB.79.214405

PACS number(s): 75.60.Ch, 75.75.+a, 75.60.Jk

\section{INTRODUCTION}

Transverse domain walls (DWs) (TDWs) in thin magnetic nanowires carry magnetostatic charge-positive for head-tohead DWs (HHDWs), negative for tail to tail DWs (TTDWs) - and this is easily accessible by, for example, detecting their direction of motion under an externally applied magnetic field of known direction. But they also carry a second degree of freedom: the chirality, i.e., the direction of rotation of the magnetization in the DW. Because the absolute moment of the transverse part is so small, it is difficult to access it experimentally. Recent studies ${ }^{1}$ showed that the strength of pinning of DWs by asymmetric traps is chirality dependent, and we recently demonstrated ${ }^{2}$ on a wide range of trap geometries that the potential disruption created by the same trap also depends on the chirality of the incoming DW. However, the type of traps we studied did not allow us to distinguish the roles of all the different characteristic parameters of the TDW in the pinning process. In particular, the role of the characteristic V shape of the DWs, ${ }^{3}$ describing the fact that a HHDW is significantly wider on the side where the DW core magnetization points out (the side where the core magnetization points in is wider in a TTDW), was difficult to isolate from the role of the relative orientation between the DW core and the magnetization in the trap. Understanding the pinning mechanism of DWs by artificial structural defects is of vital importance for novel magnetic DW logic ${ }^{4,5}$ and memory devices ${ }^{6-8}$ which use controlled DW movement in complex magnetic nanowire networks. Furthermore, the ability to control the structure of a domain wall through the geometrical dimensions of the magnetic wire allows the experimental study of fundamental physical properties of these different types of DWs. ${ }^{9-12}$ Although there have been several experimental studies reporting the ability of artificially created constrictions to pin DWs, ${ }^{10,13-15}$ and numerous spin-transfer experiments currently use such artificial defects to precisely locate and hold DWs within magnetic nanostructures, ${ }^{16,17}$ a complete understanding of how the local DW energy landscape is modified by artificial structural defects is currently lacking.

In this paper, we report the use of spatially resolved magneto-optical Kerr effect (MOKE) measurements to study experimentally the switching properties of Permalloy nanowires containing a simple model trap constituted by a Permalloy transverse arm (TA). The TA stops at the horizontal wire, forming a $\mathrm{T}$ structure (see the top of Fig. 1). Because of the shape anisotropy, the magnetization in the TA runs parallel $(\mathrm{P})$ to the TA, greatly simplifying the micromagnetic configuration inside the trap, and therefore the interaction of such traps with DWs. These T-shaped traps are found to pin DWs effectively. We measured the pinning strengths as well as the potential disruptions seen by DWs, i.e., whether DWs are pinned because they cannot escape from potential wells, or because they are unable to jump over potential barriers. The orientation of the incoming DW magnetization relative to the magnetization in the TA is found to be linked to the type of potential disruption experienced by the TDW. Potential wells are observed when the magnetization in the TA is parallel to the magnetization in the core of the TDWs, and potential barriers in the antiparallel (AP) case. Furthermore, $\mathrm{T}$ structures allow us to study separately the role of the orientation of the magnetization in the DW core relative to the magnetization in the trap and the effect of the characteristic triangular shape of TDWs. For the same parallel or antiparallel DW or trap configuration, the strength of pinning is found to depend on whether the trap is on the wide or on the narrow side of the DW. We present a numerical study of the magnetic charge distribution within TDWs which show that most of the DW charge is situated on the wide side of the DW. OOMMF (Ref. 18) micromagnetic calculations were performed which are in very good qualitative agreement with the experimental results and show that the DW shape asymmetry dependence of the pinning strength and pinning potential is a consequence of the possibility for the system to lower its demagnetizing energy by reorganizing the magnetic charge distribution in the DW within the trap. 


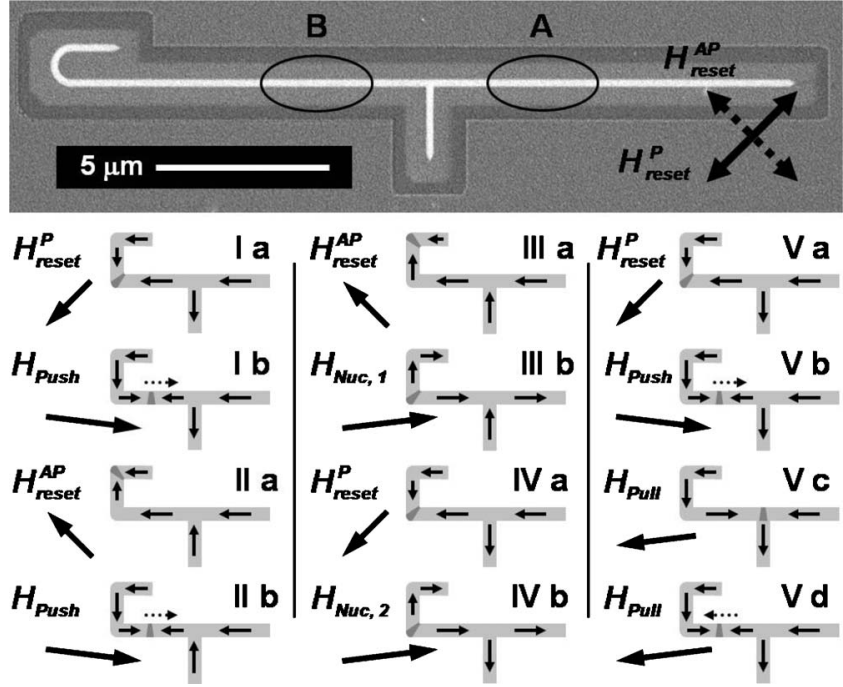

FIG. 1. Top: secondary electron image by FIB irradiation of a 200-nm-wide Permalloy structure with a T-shaped trap at its middle. Ellipses A and B show the two positions of the laser beam during MOKE measurements; the double arrows show the directions $H_{\text {reset }}^{\mathrm{P}}$ and $H_{\text {reset }}^{\mathrm{AP}}$. Bottom: schematics illustrating the field sequences used to measure the switching properties of the structures. Ia, Ib, IIa, and IIb illustrate the creation and displacement of a DW; IIIa, IIIb, IVa, and $\mathrm{IVb}$ illustrate the nucleation field measurements; and $\mathrm{Va}-\mathrm{Vd}$ illustrate the potential measurements. The large arrows indicate the direction of the external magnetic field, the narrow arrows show the direction of the magnetization in the nanostructure, and the dotted arrows show the direction of displacement of DWs.

\section{EXPERIMENTAL}

200-nm-wide structures were milled using focused 30 $\mathrm{keV} \mathrm{Ga}^{+}$ions from 7-nm-thick thermally evaporated $\mathrm{Ni}_{80} \mathrm{Fe}_{20}$ film on silicon substrate. 200-nm-wide and $2-\mu \mathrm{m}$-long perpendicular arms were patterned in the same milling process. One end of each structure was patterned into a $\mathrm{C}$ shape for DW creation. The thickness and width of our structures lie very close to the calculated stability limit between vortex and TDWs. ${ }^{3,19}$ However, although both DW types are energy minima, there is not enough thermal activation at room temperature for the TDW which is initially created to overcome the energy barrier which separates it from the vortex wall configuration. ${ }^{20}$ The perpendicular arm is either placed outside the curvature of the $\mathrm{C}$, forming a $\mathrm{T}$ structure ("out" type on the top left diagram of Fig. 2), or inside, forming an upside down T structure ("in" type on the bottom left diagram of Fig. 2); the chirality of the corner is kept the same throughout. A micrograph of a T-shaped structure is shown at the top of Fig. 1.

Each structure was analyzed using a high sensitivity MOKE magnetometer. The $\sim 5$ - $\mu$ m-diameter-focused laser spot was placed on the horizontal section of the wire on either side of the trap. A quadrupole electromagnet was used to apply $H_{x}$ and $H_{y}$ magnetic fields at a frequency of $1 \mathrm{~Hz}$ : $H_{x}$ (along the long part of the $\mathrm{C}$ shape) is a sinusoidal field, and as it reaches its maximum (and minimum) an $H_{y}$ pulse is applied producing a $45^{\circ}$ reset field pulse. Depending on the relative sign of $H_{x}$ and $H_{y}$, the resulting field is either aligned

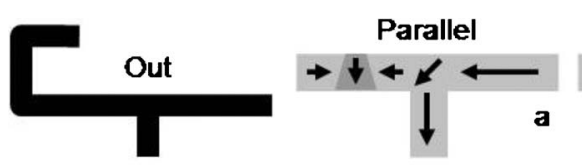

Antiparallel
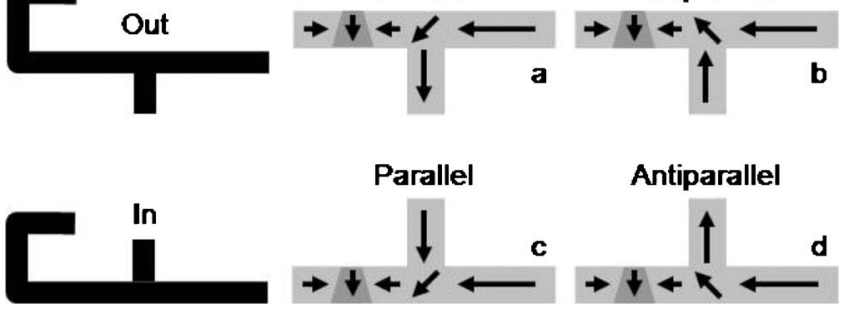

FIG. 2. Left: schematics showing the shape of the structures. One end of each structure is patterned into a $\mathrm{C}$ shape for DW creation; the TA is either patterned outside the curvature of the $\mathrm{C}$ shape (out type) or inside (in). Right: schematics illustrating the four possible DW/TA configurations. For the same out or in-type structure, the magnetization is either parallel to the TA [(a) and (c)] or antiparallel [(b) and (d)].

with the solid double arrow $H_{\text {reset }}^{\mathrm{P}}$ shown at the top of Fig. 1 or with the dotted one $H_{\text {reset. }}^{\mathrm{AP}}$. In the first case, a DW is created in the bottom corner of the $\mathrm{C}$ shape and the magnetization in the vertical arm points downward; in the second case a DW is created in the top corner and the TA magnetization points upward. $H_{x}$ and $H_{y}$ subsequently decrease together so that the resulting field direction remains approximately at $45^{\circ}$ down to a final value $H_{y}=H_{y}^{\text {off }}= \pm 10$ Oe and $H_{x}=0$. If the signs of the $y$ component of $H_{\text {reset }}$ and $H_{y}^{\text {off }}$ are the same, then the DW remains in the corner where it was created; on the contrary, it moves to the opposite corner if the signs of the $y$ component of $H_{\text {reset }}$ and $H_{y}^{\text {off }}$ are different. $H_{y}^{\text {off }}$ is sufficient to displace a DW in a simple wire but far below the field necessary to nucleate a DW and switch the TA. $H_{y}$ remains at the same $H_{y}^{\text {off }}$ value while $H_{x}$ increases in the opposite direction, causing the DW to move toward the right in whatever arm $H_{y}^{\text {off }}$ placed it initially.

\section{RESULTS}

\section{A. Switching fields}

Three types of switching fields were measured for a given nanowire structure: the propagation $H_{P}$, transmission $H_{T}$, and nucleation $H_{N}$ fields. The four field sequences (I, II, III, and IV), which test the four possible combinations between the relative signs of $H_{\text {reset }}$ and $H_{y}^{\text {off }}$ together with schematics describing the micromagnetic configuration they induce in an out-type structure, are shown at the bottom of Fig. 1. Figure 3 shows some typical MOKE hysteresis loops measured on an out-type structure using these field sequences, together with the corresponding $H_{y}$ versus $H_{x}$ curves. The relatively high level of noise in the loops is because of the extremely small volume of magnetic material, and hence magnetic moment, being detected in the experiments-typically $10^{-12}$ emu. As field sequence I is applied, a downward HHDW is created at the bottom corner, and the magnetization in the TA points downward [Fig. 1, (Ia)]; $H$ is then switched to $H_{y}^{\text {off }}=-10$ Oe (to ensure that the DW remains in the lower arm). The DW then moves toward the trap [Fig. 1, (Ib)] under the influence of $H_{\text {push }}$, the $y$ component of which remains at -10 Oe. It goes through the trap when $H_{\text {push }}$ is 


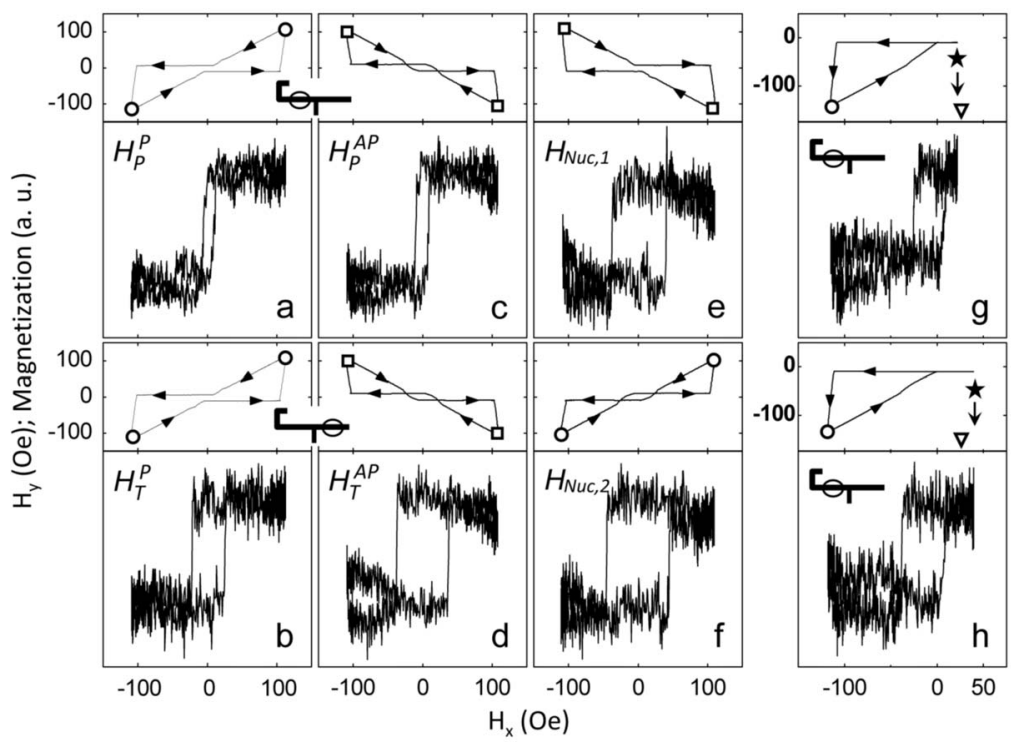

FIG. 3. MOKE-determined magnetization hysteresis loops (bottom panels) and corresponding field sequences (top panels) used to measure (a) $H_{P}^{\mathrm{P}}$, (b) $H_{T}^{\mathrm{P}}$, (c) $H_{P}^{\mathrm{AP}}$, (d) $H_{T}^{\mathrm{AP}}$, (e) $H_{\mathrm{Nuc}, 1}$, (f) $H_{\text {Nuc, },},(\mathrm{g})$, and (h) the potential profile in the $\mathrm{P}$ case. The structure has a TA situated outside the curvature of the $\mathrm{C}$ shape (out). $\bigcirc$ indicates $H_{\text {reset, }}^{\mathrm{P}}$, $\square$ indicates $H_{\text {reset }}^{\mathrm{AP}}$, the arrows labelled with $\star$ in (g) and (h) show $H_{\text {push }}$, and $\nabla$ shows $H_{T}$ as measured in $\mathrm{b}$. The schematics indicate on which part of the nanostructure the MOKE laser spot was focused. high enough and annihilates at the end of the nanowire. Unless otherwise stated, all field sequences are in two parts. The second half of the field cycle, where an upward TTDW is created and similarly pushed toward the right (not shown here), is obtained by the reversal of all the fields. Note that the V-shape asymmetry is preserved during the whole field cycle: the wide side of the DW is on the lower side for both the HHDW and the TTDW. The configurations obtained using field sequence I are referred to as $\mathrm{P}$, since the magnetization in the core of the DW is parallel to the magnetization in the TA. A schematic of the micromagnetic configuration is shown in Fig. 2(a) [the parallel configuration schematically shown in Fig. 2(c) is obtained for an in-type structure]. The corresponding MOKE hysteresis loop measured between the corner and the trap (position B at the top of Fig. 1) is presented in Fig. 3(a) for an out structure and shows a sharp transition as $H_{x}$ reaches the propagation field $H_{P}^{\mathrm{P}}$ necessary for the DW to move from the corner toward the trap, thereby reversing the magnetization in part B of the structure. When the laser spot is placed between the trap and the end of the nanowire (position A at the top of Fig. 1), a sharp transition is observed as $H_{x}$ reaches the transmission field $H_{T}^{\mathrm{P}}$ necessary for the DW to travel through the trap and reverse part A of the nanowire [Fig. 3(b)]. $H_{P}^{\mathrm{P}}$ and $H_{T}^{\mathrm{P} \text {,out }}$ were measured at $6 \pm 1$ Oe and $25 \pm 3$ Oe, respectively $\left(H_{T}^{\mathrm{P} \text {,in }}\right.$ was measured at $9 \pm 2 \mathrm{Oe})$. The quoted error is the standard deviation obtained by measuring the switching properties of five different structures.

The nucleation field is the switching field measured when no DW is initially present. When using field sequence III, a $\mathrm{DW}$ is created at the top corner when $H_{\text {reset }}^{\mathrm{AP}}$ is applied and the bottom part of the structure is aligned clockwise [Fig. 1(IIIa) $]$. Then $H$ decreases, leaving a $y$ offset $H_{y}^{\text {off }}$ in the same direction as $H_{\text {reset }}^{\mathrm{AP}}$, which ensures that the initial DW remains in the top arm. As $H_{x}$ is reversed, the first DW moves toward the free end of the upper arm and annihilates, while a new DW nucleates in the bottom part which reverses it. The corresponding hysteresis loop for the out structure is shown in Fig. 3(e). $H_{\mathrm{Nuc}, 1}$ was measured at $38 \pm 5$ Oe. When using field sequence IV, a DW is created at the bottom corner using $H_{\text {reset }}^{\mathrm{P}}$. Then $H$ decreases, leaving a $y$ offset $H_{y}^{\text {off }}$ in the direction opposite to $H_{\text {reset }}^{\mathrm{P}}$, which ensures that the initial DW is pushed to the upper corner. As $H_{x}$ is reversed, the DW moves toward the free end of the upper arm and annihilates, while a new DW nucleates in the bottom part of the wire which reverses it. The corresponding hysteresis loop is presented in Fig. 3(f). $H_{\mathrm{Nuc}, 2}$ was measured at $43 \pm 3$ Oe. The fact that two different nucleation fields are measured in the same structure simply reflects the different configurations at the trap during the two measurements. The field applied to measure $H_{\mathrm{Nuc}, 2}$ has a $y$ component in the opposite direction to the magnetization in the TA, whereas the $y$ component of the field used to measure $H_{\mathrm{Nuc}, 1}$ is in the same direction. C-shape structures without a TA switch at 57 Oe using both field sequences, suggesting that the junction with the TA is a nucleation center.

In the case of the field sequence shown in Fig. 1(II), an upward HHDW is created at the top corner of the $\mathrm{C}$ shape using $H_{\text {reset }}^{\mathrm{AP}}$, and the TA points upward [Fig. 1(IIa)]. $H$ then decreases, leaving a $y$ offset $H_{y}^{\text {off }}$ in the opposite direction to $H_{\text {reset }}^{\mathrm{AP}}$, which causes the DW to move from the top to the bottom corner, but leaves the magnetization in the vertical arm still pointing up [Fig. 1(IIb)]. In that case the DW, which initially points up, travels with its core magnetization pointing outward, to eventually point down when in the lower arm. This case is referred to as AP since the magnetization in the core of the DW is antiparallel to the magnetization in the TA. A schematic of the micromagnetic configuration is shown in Fig. 2(b) [the AP configuration schematically shown in Fig. 2(d) is obtained for an in-type structure]. The loop measured between the corner and the trap using field sequence II [Fig. 3(c)] shows a sharp transition at $H_{P}^{\mathrm{AP}}$ $=6.4 \pm 2 \mathrm{Oe}$, confirming that the $\mathrm{DW}$ is indeed traveling from the top to the bottom part of the structure. When the laser spot is placed on the other side of the vertical arm, a sharp transition occurs at $H_{T}^{\mathrm{AP} \text {,out }}=33 \pm 3$ Oe [Fig. 3(d)],

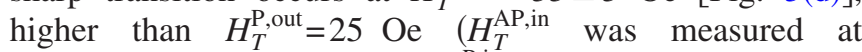
$42 \pm 4$ Oe, much higher than $H_{T}^{\mathrm{P} \text {,in }}=9$ Oe). 


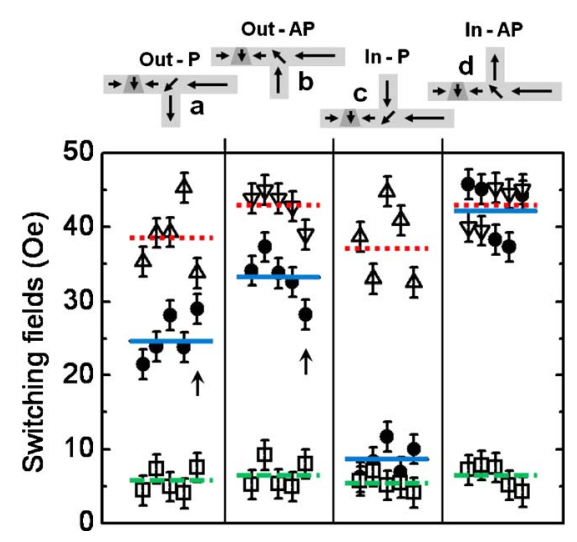

FIG. 4. (Color online) Top: the four possible orientation configurations for a TDW and a TA. (a) and (c) are P configurations; (b) and (d) are AP configurations. Note how within the same P or AP configurations the TA interacts with either side of the TDW, depending on whether the structure is out type [(a) and (b): the trap is on the wide side of the DW] or in type [(c) and (d): the trap is on the narrow side of the DW]. Bottom: associated switching fields measured on five structures of each configuration. $\square: H_{P}^{\mathrm{P}, \mathrm{AP}}, \triangle$ : $H_{\mathrm{Nuc}, 1}, \nabla: H_{\mathrm{Nuc}, 2}$, and $\bullet: H_{T}^{\mathrm{P}, \mathrm{AP}}$. Green dashed line: $H_{P}$ group average, blue solid line: $H_{T}$ group average, and red dotted line: $H_{N}$ group average.

The lower part of Fig. 4 summarizes the switching fields measured on ten structures (five in and five out). Each structure has been studied both in the P and AP configurations and therefore appears twice on the diagram. The order in which the structures appear is the same in each P and AP blocks (for example, the two sets of data marked with an arrow have been measured on the same structure). $H_{T}^{\mathrm{P}, \mathrm{AP}}$ (full circles) is plotted together with $H_{P}$ (squares) and $H_{\text {Nuc, } 1,2}$ (triangles). The dependence of the transmission field on the configuration is clearly seen. For the same in or out structure, the pinning field in the AP case is systematically higher than the pinning field in the $\mathrm{P}$ case. Only in the case of the structure marked with an arrow is the transmission field the same in both $\mathrm{P}$ and AP cases. For in-type structures, for instance, the pinning field in the $\mathrm{P}$ configuration is barely higher than the propagation field, whereas the pinning field in the AP configuration is so high that part $\mathrm{A}$ of the structure cannot switch before a field on the order of $H_{\mathrm{Nuc}, 2}$ is applied. This dependence of the transmission field of a given DW on the TA orientation provides a mechanism for a switchable DW gate device. ${ }^{21}$ More surprising is the fact that for the same $\mathrm{P}$ or AP relative orientation, whether the TA is placed inside or outside the curvature of the $\mathrm{C}$ shape matters. For the $\mathrm{P}$ configuration for example, $H_{T}^{\mathrm{P}}$ is much lower in the in case (c) than in the out case (a), i.e., the DW is pinned more strongly when the TA is on the wide side of the DW. This complex dependence of the transmission field on the DW chirality provides a basis for DW filtering devices, where applied fields control the transmission of DWs. More precisely, if a DW in the in P configuration travels past the TA with minor disruption, a DW of the opposite chirality finds itself in the out AP configuration and is strongly pinned by the same TA. Similarly, a DW in the out $\mathrm{P}$ configuration is pinned more strongly than a DW of the opposite chirality (in the in AP

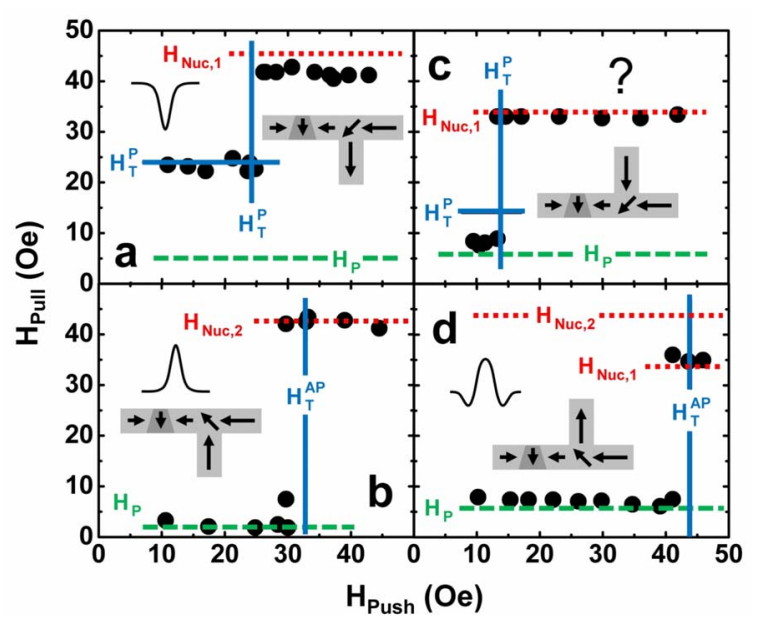

FIG. 5. (Color online) $H_{\text {pull }}$ vs $H_{\text {push }}$ graphs for [(a) and (b)] an out and $[(\mathrm{c})$ and (d)] an in structure, both in the [(a) and (c)] P and [(b) and (d)] AP configurations. A schematic of the potential profile is inserted in each case, together with a schematic of the DW/trap configuration. Green dashed line: $H_{P}$; blue solid line: $H_{T}$; and red dotted line: $H_{N}$.

configuration), also demonstrating chirality filtering although for a smaller range of fields.

\section{B. Potential measurements}

Potential measurements ${ }^{2}$ were also performed on some of the structures using the field sequence described in Fig. 1(V). In that case, a DW is created at either corner of the structure using $H_{\text {reset }}[$ Fig. $1(\mathrm{Va})]$ and pushed toward the trap under the appropriate $H_{y}^{\text {off }}$, controlling the maximum value of $H_{\text {push }}$ [Fig. 1(Vb)]. The horizontal component of the field is then reversed to try and pull the initial DW back from the trap to the corner [Fig. 1(Vc and $\mathrm{Vd})]$. By measuring the field $H_{\text {pull }}$ necessary to pull the DW back to the corner of the structure as a function of $H_{\text {push }}$, the potential profile experienced by the DW can be experimentally measured. Two hysteresis loops are presented in Figs. 3(g) and 3(h) in the case of an out structure. In both cases, the positive transition corresponds to $H_{P}^{\mathrm{P}}$, which is the field necessary to move the DW from the corner toward the trap and reverse part B of the structure. Figure $3(\mathrm{~g})$ shows the $H_{\text {push }}<H_{T}^{\mathrm{P}}$ case, where a field also equal to $H_{T}^{\mathrm{P}}$ is required to pull the DW back out of the trap; Fig. 3(h) shows the $H_{\text {push }}>H_{T}^{\mathrm{P}}$ case where the DW has gone past the trap and a field equal to $H_{\mathrm{Nuc}, 1}$ has to be applied to reverse the magnetization. Figure $3(\mathrm{~g})$ is characteristic of a potential well. Figure 5 shows $H_{\text {pull }}$ versus $H_{\text {push }}$ for all four configurations. The lines indicate the characteristic switching fields for these particular structures. The out $\mathrm{P}$ case is displayed in Fig. 5(a) and shows a simple symmetric potential well where the DW can be pulled back from the trap area with a field equal to $H_{T}^{\mathrm{P}}$ as long as $H_{\text {push }}<H_{T}^{\mathrm{P}}$. The AP case presented in Fig. 5(b) shows a simple barrier where a reverse field as low as $H_{P}$ is sufficient to pull the DW back from the trap. The in P case shown in Fig. 5(c) is not clear. The low $H_{\text {push }}<H_{T}^{\mathrm{P}}$ regime where the DW can be pulled back with a field significantly lower than $H_{T}^{\mathrm{P}}$, but only 
slightly higher than $H_{P}$, can be interpreted as the signature of a barrier with side wells, as well as a double barrier with a shallower well in between. This case will be discussed later in the light of the micromagnetic simulations. The AP case presented in Fig. 5(d) shows that the DW experiences a barrier. The data are also compatible with the existence of side wells (cf. the $H_{\text {push }}<H_{T}^{\mathrm{AP}}$ regime where the DW can be pulled back with a field only slightly higher than $H_{P}$ ). As previously mentioned, $H_{T}^{\mathrm{AP}}$ is similar to $H_{\mathrm{Nuc}, 2}$; it is not clear therefore whether the original DW transmits or whether a new DW nucleates on the other side of the trap. The fact that the high-field $H_{\text {push }}>H_{T}^{\mathrm{AP}}$ regime, where the initial DW has traveled past the trap and a new DW has to nucleate in order to switch the nanowire, corresponds to $H_{\mathrm{Nuc}, 1}$ rather than $H_{\mathrm{Nuc}, 2}$, will also be discussed later. In brief, these measurements confirm our earlier finding ${ }^{2}$ that the chirality of a DW not only affects how strongly it will be pinned by the same trap but it also influences the type of potential landscape a DW experiences as it approaches a pinning site. They also confirm the link between the type of potential disruption observed and the relative orientation of the magnetization inside the core of the DW and the magnetization in the trap. Barriers are systematically measured in the AP case, and a clear well is measured in one $\mathrm{P}$ case; the other $\mathrm{P}$ configuration is not so evident but is also compatible with a well. Furthermore, these results show very explicitly the effect of the V-shape structure of the TDW. The pinning strength is significantly higher if the trap is placed on the wide side of the V.

\section{MICROMAGNETIC SIMULATIONS}

In order to understand our experimental results, we have performed micromagnetic simulations using the OOMMF package $^{18} \quad\left(M_{S}=800 \times 10^{3} \mathrm{~A} / \mathrm{m}, A=13 \times 10^{-12} \mathrm{~J} / \mathrm{m}\right.$, and $3.5 \times 3.5 \times 7 \mathrm{~nm}^{3}$ cell size). The simulations were performed quasistatically, with $\alpha$ set to 0.5 to speed up the calculations. As previously mentioned, T-shaped traps allow to isolate and probe the role of the DW V-shape asymmetry, and therefore the role of the inhomogeneous magnetic charge distribution in the pinning process. Figure 6(a) shows the calculated micromagnetic configuration of a HHDW in a 200-nm-wide and 7-nm-thick Permalloy nanowire. The characteristic V shape is clearly seen, with an upper wide side where the magnetic moments rotate rather slowly across the DW width and a lower narrow side where the magnetic moments rotate more rapidly. The corresponding magnetic charge density defined as $\rho=-\mu_{0} \vec{\nabla} \vec{M}$ is mapped out in Fig. 6(b); the contrast ranges from white (negative charge density) to black (positive). As expected for a HHDW, the overall charge is positive. The charge distribution follows the V shape of the DW, and the charge density is higher where the magnetic moments rotate rapidly (lower side of the DW). Two high charge density lines are observed on either side of the DW along the edges of the wire: a positive line along the upper wide side and a negative one along the lower narrow side. The main contribution to these surface charge density lines comes from the high $\partial M_{y} / \partial y$ term at the sample edges where the magnetic moments are perpendicular to the surface. It is

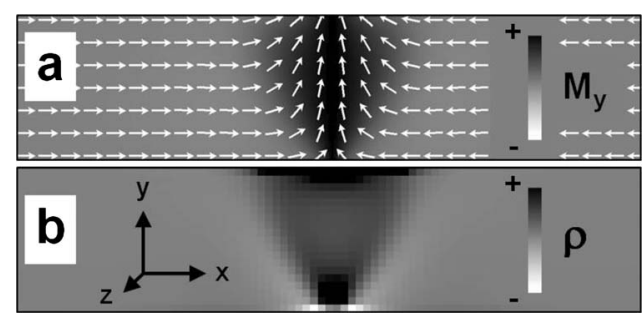

FIG. 6. (a) Micromagnetic configuration and (b) magnetic charge distribution associated with a HHDW in a 200-nm-wide and 7-nm-thick Permalloy nanowire. The contrast in (a) shows the vertical component of the magnetization and goes from white (down) to black (up). The white arrows indicate the direction of the magnetization. The contrast in (b) goes from white for negative charge to black for positive charge. The bulk of the DW is made of positive volume charge, with a long strong positive surface charge line on the wide top edge of the DW, and a shorter strong negative surface charge along the narrow edge of the DW.

positive where the magnetization points toward the outside of the wire, negative when it points toward the inside. The total magnetic charge contained in a given volume is the volume integral of the magnetic charge density. Numerically integrating over the charge distribution shown in Fig. 6(b), we find that all the DW charge $\left(2 Q=2 \mu_{0} M_{S} S\right.$, where $S$ is the cross-sectional area of the nanowire) is contained in a $\left(L_{x}\right.$ $=250 \mathrm{~nm}$ wide $) \times\left(L_{y}=200 \mathrm{~nm}\right.$ high $) \times\left(L_{z}=7 \mathrm{~nm}\right.$ deep $)$ box centered on the DW. When fixing $L_{x}$ at $250 \mathrm{~nm}$, we find that $75 \%$ of the total DW charge is contained within the upper $70 \mathrm{~nm}$ of the DW (one third of the wire width) and that the upper $20 \mathrm{~nm}$ of the DW (one tenth of the wire width) contains half of the DW charge. The charge contained within a $250 \mathrm{~nm}$ long line along the narrow side of the DW is $-0.2 Q$, and it is cancelled out by the positive volume charge contained in a box adjacent to the surface and about $50 \mathrm{~nm}$ high, i.e., the lower $50 \mathrm{~nm}$ of the DW contains no net magnetic charge. To summarize, the magnetic charge associated with a TDW is asymmetrically distributed within the DW and most of it is situated on the wide side of the DW.

Having determined the morphology of a TDW, we now turn to the simulated transmission, nucleation, and potential experiments. Each of the three arms of the T structures was $1 \mu \mathrm{m}$ long and $200 \mathrm{~nm}$ wide; the whole structure was $7 \mathrm{~nm}$ thick. All free ends were shaped into a point in order to avoid domain nucleation at the extremities. A DW of either chirality was artificially placed next to an upward or downward TA and left to relax under $H_{y}^{\text {off }}$ (to which the minimum horizontal field required to prevent the DW from being repelled by the trap and annihilate at the free end of the structure was added if needed), after which $H_{x}$ was increased in 5 Oe steps in order to push the DW toward the trap. The calculations were performed with $H_{y}^{\text {off }}= \pm 10$ and \pm 65 Oe. All simulated switching fields are shown in Table I. Quantitative agreement is not expected between experiments performed at room temperature and simulations performed at $0 \mathrm{~K},{ }^{22}$ and the experimental switching fields are on average $70 \%$ lower than the calculated ones. Nevertheless, qualitative agreement can still be expected. The simulations agree fairly well with the experiments in the $\mathrm{P}$ transmission cases, where the in configu- 
TABLE I. Summary of the switching field $H_{T}^{\mathrm{P}, \mathrm{AP}}, H_{\mathrm{Nuc}, 1,2}$, and $H_{\text {pull }}^{\mathrm{P}, \mathrm{AP}}$ values obtained with the simulations. The values are quoted \pm 2.5 Oe. $H_{\text {pull }}^{\mathrm{P}, \mathrm{AP}}$ are presented as $H_{\text {pull }}^{\mathrm{P}, \mathrm{AP}, 1} / H_{\text {pull }}^{\mathrm{P}, \mathrm{AP}, 2}$, where $H_{\text {pull }}^{\mathrm{P}, \mathrm{AP}, 1}$ is calculated for $H_{\text {push }}<H_{T}^{\mathrm{P}, \mathrm{AP}}$ and $H_{\text {pull }}^{\mathrm{P}, \mathrm{AP}, 2}$ for $H_{\text {push }}>H_{T}^{\mathrm{P}, \mathrm{AP}}$.

\begin{tabular}{|c|c|c|c|c|c|c|}
\hline \multicolumn{7}{|c|}{$H_{y}^{\mathrm{off}}=10 \mathrm{Oe}$} \\
\hline Out & 82.5 & $142.5^{\mathrm{a}}$ & 167.5 & 177.5 & $82.5 / 167.5$ & $0 / 17.5$ \\
\hline In & 22.5 & $122.5^{\mathrm{b}}$ & 167.5 & 177.5 & $0^{\mathrm{c}}-22.5 / 167.5$ & $7.5 / 167.5$ \\
\hline \multicolumn{7}{|c|}{$H_{y}^{\mathrm{off}}=65 \mathrm{Oe}$} \\
\hline Out & 67.5 & $177.5^{\mathrm{a}}$ & 132.5 & $202.5^{\mathrm{d}}$ & $67.5 / 132.5$ & $0 / 12.5$ \\
\hline In & 17.5 & $87.5^{\mathrm{b}}$ & 132.5 & $207.5^{\mathrm{e}}$ & $0^{f}-17.5 / 132.5$ & $7.5 / 132.5$ \\
\hline
\end{tabular}

aAlthough the structure has reversed, the initial DW is still trapped in front of the oppositely magnetized trap, forming a $360^{\circ} \mathrm{DW}$.

${ }^{\mathrm{b}}$ The initial DW transmits through the oppositely magnetized trap, creating a second DW in the vertical arm of the structure which reverses it in the presence of a $y$ offset.

${ }^{c}$ The main potential disruption is a well, but side barriers are also observed for $H_{\text {push }}<17.5$ Oe.

${ }^{\mathrm{d}}$ The transverse arm switches as well, through the following process. A DW with a core magnetization antiparallel to the TA and with a charge on the opposite side as the TA nucleates in the horizontal arm and switches it; when at the junction with the TA, this DW behaves the same way as in b, creating a second DW in the TA which reverses it.

e The magnetization reversal takes place in the horizontal arms on both sides of the junction, forming a $360^{\circ}$ DWs on either side of it.

fSame as c but for $H_{\text {push }}<12.5$ Oe.

ration is systematically found to pin less than the out configuration (82.5 Oe for the out configuration vs 22.5 Oe for the in configuration for 10 Oe offset). $H_{\mathrm{Nuc}, 1}$ is also systematically calculated at a lower value than $H_{\mathrm{Nuc}, 2}(167.5$ Oe for $H_{\mathrm{Nuc}, 1}$ vs 177.5 for $H_{\mathrm{Nuc}, 2}$ with 10 Oe offset). The calculations show that a too large $y$ offset opposed to the direction of the magnetization in the TA causes the junction between the TA and the horizontal wire to cease being a nucleation center, leading to a complex reversal pattern (see Table I). For an offset of 65 Oe, in the in case, for instance, two DWs with core magnetization pointing in the opposite direction to the TA nucleate on either side of the TA, resulting in the formation of a $360^{\circ} \mathrm{DW}$ on either side of it. On the contrary, a higher $y$ offset in the same direction as the magnetization in the TA eases the nucleation process at the junction and therefore decreases $H_{\mathrm{Nuc}, 1}$ (167.5 Oe for 10 Oe offset and 132.5 Oe for 65 Oe offset).

The calculated potential measurements in the out $\mathrm{P}$ case [Fig. 7(a)] are also in excellent qualitative agreement with experiments where a simple well is observed for both values of the $y$ offset $\left(H_{\text {pull }}^{\mathrm{P}}=H_{T}^{\mathrm{P}}\right.$ for $\left.H_{\text {push }}<H_{T}^{\mathrm{P}}\right)$. In that case, the DW simply merges with the TA and a field of the same amplitude is required to further propagate the DW or to pull it back. We estimated the energy of a DW in a given configuration by calculating the difference, in zero field, between the energy of the same structure with and without a DW. In the latter out P case, the energy of the DW decreases by $32 \mathrm{eV}$ when it merges with the TA. This decrease in energy is mainly due to a reduction in demagnetizing energy; the decrease in the exchange energy is only $2.5 \mathrm{eV}$. Whether the DW is inside or outside the junction, the total magnetic charge contained in a box enclosing the DW and the junction is $Q=\mu_{0} M_{S} S$ [cf. Fig. 2(a)]. When the DW is outside the junction, this $+Q$ charge is distributed between the DW $(+2 Q)$ and the junction $(-Q)$. When the DW is inside the junction then all the $+Q$ charge is contained in the junction. The magnetostatic energy of the system in that case is therefore lower by an amount on the order of the magnetostatic energy associated with the DW. Furthermore, the attractive potential experienced by the positively charged DW in the vicinity of the negatively charged junction has been observed in the simulations.

The in $\mathrm{P}$ case [Fig. 7(c)] is slightly more complex. In agreement with the experiments, the pinning field is calculated at a lower value than for the out $\mathrm{P}$ configuration. The potential profile, however, could not be clearly resolved experimentally; the simulations show that the DW first experiences a barrier (for $H_{\text {push }}<17.5$ Oe when $H_{y}^{\text {off }}=10 \mathrm{Oe}$ and for $H_{\text {push }}<12.5$ Oe when $H_{y}^{\text {off }}=65 \mathrm{Oe}$ ), as the $+2 Q$ magnetic charge carried by the DW is repelled by the $+Q$ charge present at the junction [cf. Fig. 2(c)]. If the field is increased further, the DW falls into a central well as it merges with the TA, from which it can only escape for $H_{\text {push }}>22.5$ Oe when $H_{y}^{\text {off }}=10$ Oe (and for $H_{\text {push }}>17.5$ Oe when $H_{y}^{\text {off }}=65 \mathrm{Oe}$ ). Under zero field, the energy of the DW in this case is $3.1 \mathrm{eV}$ higher than when the DW sits outside the TA. This central well is only a local-energy minimum for the DW. Although the exchange energy is lower for the merged configuration, the demagnetizing energy is greater, so that the total energy is increased. Whether the DW is inside or outside the junction, the total magnetic charge contained in a box enclosing the DW and the junction is $+3 Q$. The existence of the central well is due to the magnetic charge reorganization which occurs as the DW merges with the TA. When this happens, the $-0.2 Q$ negative surface charge present at the narrow side of the DW disappears, allowing a decrease in the positive 

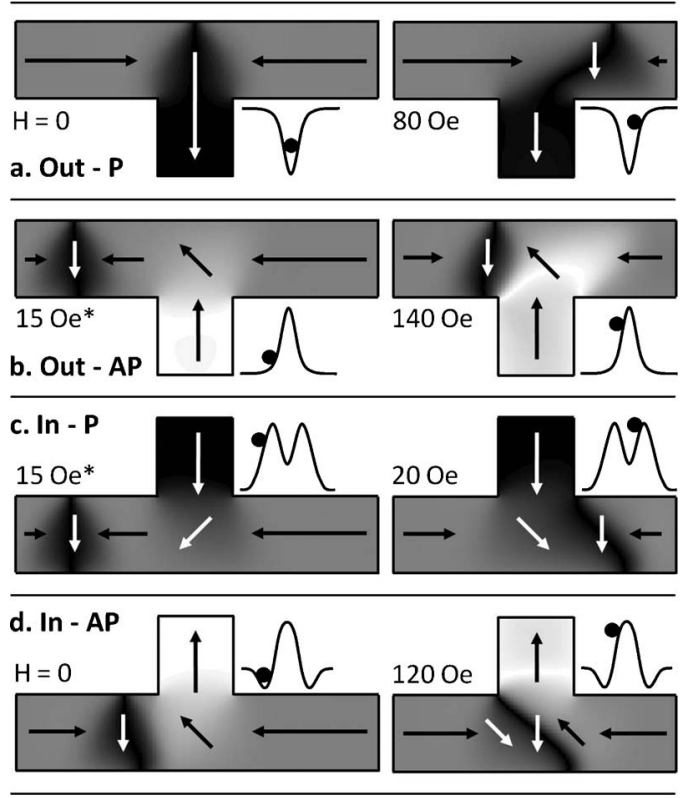

FIG. 7. Micromagnetic configurations showing a TDW pinned in each of the four DW/TA configurations, under various applied magnetic fields. The contrast shows the vertical component of the magnetization and goes from white (up) to black (down). The $*$ in $\mathrm{b}$ and $\mathrm{c}$ indicates that these configurations are not equilibrium ones; the positively charged DW was slowly drifting away from the positively charged junction under the influence of the long-range magnetostatic repulsion, even though a 15 Oe field was applied. The schematics indicate what part of the potential profile the DW is probing.

charge contained in the junction of the same amount, so that the total charge is maintained at $+3 Q$. Although the merged configuration has a higher energy than the configuration where the $+2 Q \mathrm{DW}$ is outside of the $+Q$ junction and far from it, it has a lower energy than the configuration where the DW is outside of the junction but sufficiently close to it that-because of the existence of the $-0.2 Q$ surface charge on the narrow side of the DW-the overall positive charge present at the junction is higher than $+3 Q$. Note that the fact that the field necessary for the DW to escape the central well is higher than the field necessary for the DW to go over the side barrier and enter the central well in the first place is compatible with the central well only being a local-energy minimum, as the absolute value of the transmission field is not an indication of the depth of the potential, rather a measurement of its steepness. The applied field $H$ adds a linear term to the pinning potential $U_{\text {pinning }}$ : when the DW moves a distance $\delta x$ in a wire of cross section $S$, the change in Zeeman energy is $\delta U_{Z}=-2 M_{S} S H \delta x=-K H \delta x$. The depinning condition $\left(\partial U_{\text {total }} / \partial x\right)<0$ is therefore satisfied for a transmission field $H_{T}$ equal to $\left.\left[(1 / K) \partial U_{\text {pinning }} / \partial x\right]\right|_{\max }$. As previously mentioned, the experimental data are compatible with the calculated double barrier.

The size of the trap is known to influence the pinning strength $^{23}$ in the case of constrictions. In order to rule out the possibility that the difference in pinning strength between the out $\mathrm{P}$ case and the in $\mathrm{P}$ case might be solely due to the ratio between the width of the trap and the width of the DW on the
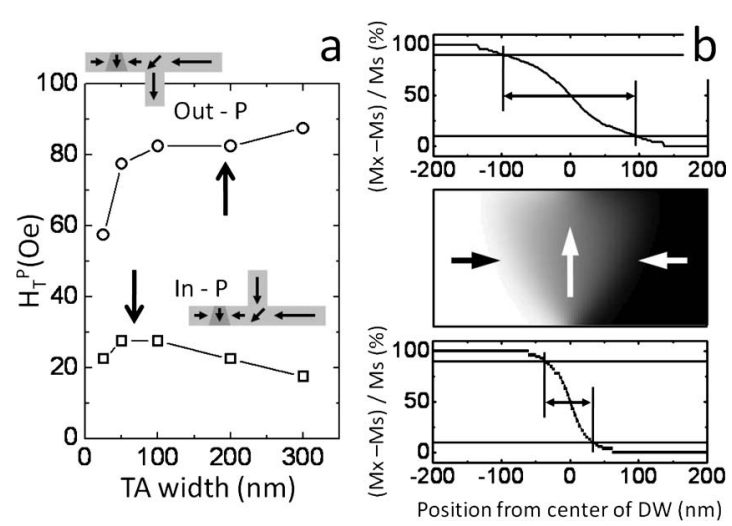

FIG. 8. (a) Transmission fields of both $\mathrm{P}$ configurations as a function of TA width. Circles: out P; squares: in P. A 10 Oe $y$-offset field was also applied. The two arrows indicate the width of the side of the DW which interacts with the TA. (b) Measurement of the widths of the wide (top) and narrow (bottom) sides of a TDW in a 200-nm-wide wire extracted from the calculated micromagnetic configuration [middle: the gray level value is proportional to the normalized horizontal component of the magnetization $\left(M_{x}\right.$ $\left.\left.-M_{S}\right) / M_{S}\right] .\left(M_{x}-M_{S}\right) / M_{S}$ along both nanowire edges is plotted as a function of the position across the DW. 10-90\% measurements indicate that the narrow side of the DW is $66 \mathrm{~nm}$ wide and the wide side is $192 \mathrm{~nm}$ wide.

side of the trap, we have performed the same transmission field simulations with varying TA widths. The results are displayed in Fig. 8(a) for $H_{y}^{\text {off }}=10$ Oe. The pinning strength does indeed depend on the TA width, but stronger pinning is always observed when the wide side of the DW is on the side of the trap. Figure 8(b) shows the measurement of the width of the DW along its wide (top) and narrow (bottom) sides. The normalized horizontal component of the magnetization along the top and bottom edge cells is plotted as a function of the position from the center of the DW. $\left(M_{x}-M_{S}\right) / M_{S}$ undergoes $80 \%$ of its total change over a distance of $192 \mathrm{~nm}$ along the wide edge of the DW and of $66 \mathrm{~nm}$ along the narrow edge. These two characteristic distances are indicated with arrows in Fig. 8(a), showing that the observed effect cannot be solely explained by the variation in the DW width. In the out case (circles), tuning the TA width to the narrow side width (arrow at $66 \mathrm{~nm}$ ) scarcely lowers the transmission field ( 80 Oe instead of 82.5 when the TA width is on the order of the wide side width arrow at $192 \mathrm{~nm}$ ).

In both AP cases, the transmission of the DW requires the energetically costly nucleation of a new DW in the TA and barriers are found as main potential disruptions. A DW in the in AP case [Fig. 7(d)] is calculated to experience side wells, as it takes 7.5 Oe to pull the DW back from the TA for $H_{\text {push }}<H_{T}^{\mathrm{AP}}$ for both values of the $y$ offset. The experimental results are compatible with the existence of side wells but the experimental resolution is not sufficient to directly affirm it. The existence of the side wells can be explained by the attractive interaction between the $+2 Q$ charge carried by the DW and $-Q$ charge present in the junction [cf. Fig. 2(d)]. As the field increases, the simulations show that the wide side of the DW, which carries the majority of the charge of the DW, keeps traveling toward the right while the narrow side re- 
mains in front of the junction, deforming the DW until it fully extends above the TA [see the right-hand side of Fig. 7(d)], creating a new DW in the vertical arm which switches it and allows the initial DW to travel past the trap. This is calculated to happen at a significantly lower field than $H_{\mathrm{Nuc}, 2}$ (it happens at $H_{T}^{\mathrm{AP}}=122.5$ Oe while $H_{\mathrm{Nuc}, 2}=177.5$ Oe under $10 \mathrm{Oe}$ offset and at $H_{T}^{\mathrm{AP}}=87.5 \mathrm{Oe}$ while $H_{\mathrm{Nuc}, 2}=207.5 \mathrm{Oe}$ under 65 Oe offset), in contrast with the experiments. However, as $H_{\text {push }}>H_{T}^{\mathrm{AP}}$, it was previously noted in the experiments that a field equal to $H_{\mathrm{Nuc}, 1}$ was required to switch the left-hand side of the structure rather than $H_{\mathrm{Nuc}, 2}$. This is explained by the switching of the TA observed in the simulations. As the field is reversed, the TA now points in the same direction as $H_{y}^{\text {off }}$, causing the left-hand side to switch at $H_{\mathrm{Nuc}, 1}$ rather than at $H_{\mathrm{Nuc}, 2}$.

Finally, in the out AP configuration [Fig. 7(b)], the simulations show that the DW remains in front of the oppositely magnetized TA while a new DW nucleates on the other side. The presence of the original DW is found to lower the nucleation field as this process happens at a lower field than $H_{\mathrm{Nuc}, 2}$ (it happens at $H_{T}^{\mathrm{AP}}=142.5$ Oe while $H_{\mathrm{Nuc}, 2}=177.5$ Oe under $10 \mathrm{Oe}$ offset and at $H_{T}^{\mathrm{AP}}=177.5 \mathrm{Oe}$ while $H_{\mathrm{Nuc}, 2}$ $=202.5$ Oe under 65 Oe offset). This agrees with transmission experiments where the right-hand side of the structure switches for a field on average 10 Oe lower than $H_{\mathrm{Nuc}, 2}$. The DW experiences a barrier as its $+2 Q$ magnetic charge is pushed toward the $+Q$ charge present at the junction as long as the right-hand side of the structure has not switched [cf. Fig. 2(b)]. This agrees with the experimental results. Then as the field is increased further and the right-hand side has reversed through the nucleation of a new DW, the initial DW is calculated to experience a shallow well due to the fact that the charge present at the junction has changed sign when the right arm has reversed (pull fields of 17.5 Oe under 10 Oe offset and 12.5 Oe under 65 Oe offset are measured). This was not observed experimentally, where a field equal to the nucleation field was necessary to switch the left-hand side of the structure, showing that the initial DW had transmitted. However, in order for the initial DW to transmit, the TA must somehow switch. One would therefore expect to measure $H_{\mathrm{Nuc}, 1}$ for $H_{\text {push }}>H_{T}^{\mathrm{AP}}$ rather than $H_{\mathrm{Nuc}, 2}$. For the structure of Figs. 5(a) and 5(b), $H_{\mathrm{Nuc}, 1}$ is too close to $H_{\mathrm{Nuc}, 2}$ to verify this, and of the other two out structures for which the potential was measured, one behaved according to this scenario, and one clearly showed switching at $H_{\mathrm{Nuc}, 2}$ rather than $H_{\mathrm{Nuc}, 1}$. This remains to be understood.

\section{EFFECT OF FIB PATTERNING}

Focused ion beam (FIB) milling is a very powerful tool for rapid direct prototyping of nanostructures. However, it is well known that the ion-induced intermixing of atoms at the interfaces can dramatically affect the properties of thin magnetic multilayers ${ }^{24,25}$ or capped films, ${ }^{26}$ even at doses so low that no etching occurs. In the case of single and noncapped magnetic layers, ion irradiation has two effects. The first one is to reduce the thickness of the magnetic active layer due to direct etching of the magnetic film and intermixing with the substrate atoms; the second effect is to reduce the layer's saturation magnetization due to ion implantation and radiation-induced damage formation. ${ }^{27,28}$ These cause a more gradual quenching of the layer's magnetic properties than in the case of multilayers, although faster than would be expected from pure etching only. The typical dose used to pattern our structures was around $5 \times 10^{15}$ ions $/ \mathrm{cm}^{2}$, just sufficient for complete etching of the magnetic layer. FIBs are known to have a high-intensity Gaussian central part, which transitions to exponential tails 2 to 3 orders of magnitude below the maximum value. ${ }^{29}$ Approximating the $10 \mathrm{pA}$ beam we used with a Gaussian of $10 \mathrm{~nm}$ full width at half maximum, the dose is reduced by a factor of 10 at a distance of 20 $\mathrm{nm}$ from the center of the beam. According to Ref. 28, this should result in a gradual reduction in the layer's magnetic moment proportional to the dose. It is not clear what the effect should be. The structures should appear narrower that they really are and therefore have higher switching fields, but the gradual sloping of the edges should have the reverse effect. $^{30}$ We have simulated the effect of reducing the magnetic moment around the edge of the structure by changing the edge thickness profile from a sharp step to a gradual linear slope, going from zero to maximum thickness over a distance of $20 \mathrm{~nm}$. Less than $10 \%$ change in the transmission field was observed, apart from the in $\mathrm{P}$ case for which the transmission field is reduced to $12.5 \mathrm{Oe}$ (instead of $22.5 \mathrm{Oe}$ ). However, the pinning mechanism remains the same.

\section{SUMMARY}

In conclusion, we have performed MOKE measurements and micromagnetic simulations on 7-nm-thick and 200-nmwide Permalloy nanowires in order to study the pinning of TDWs by transverse arms forming T structures with the DW conduit. The micromagnetic configuration in the trap (either up or down) was controlled using external magnetic fields, and the chirality of the DW with respect to the trap was controlled by patterning the latter either on the outer or on the inner part of the structure. These $\mathrm{T}$ structures allowed us to study separately the influence of the different DW parameters (core orientation and magnetic charge distribution) on the pinning process. Two types of measurements were performed: switching field measurements (propagation, nucleation, and transmission) and potential profile measurements. We have shown that the pinning strength as well as the type of potential disruption experienced by a DW depends on its chirality. The same trap can act as a potential well or as a potential barrier, depending on the chirality of the incoming DW. We show that (the energy of a DW being dominated by the demagnetizing term) two factors contribute to its interaction with a TA. The first interaction is the magnetostatic interaction between the charge carried by the DW and charge present at the junction. The junction having three arms, it has to possess a positive or negative net charge which either attracts or repels the charged DW. This term is observed as side barriers or side wells around the main potential barrier or well. The main potential disruption is seen to depend on the relative orientation of the magnetization in the core of the DW and the magnetization in the trap. In order for the junction with the TA to be a stable position for the DW (and 
therefore to constitute a potential well), the DW has to be $\mathrm{P}$ to the magnetization in the trap. The formation of a new DW in the TA, which is required for a DW to sit at an oppositely magnetized TA, is very costly and results in potential barriers in the AP cases. Within the two possible $\mathrm{P}$ configurations, one is seen to pin DWs more strongly and corresponds to the arrangement where the TA is patterned on the side of the DW which carries the majority of the magnetic charge of the DW (wide side of the V-shaped DW). In that case, the merging of the DW with the TA results in a drastic lowering of the demagnetizing energy of the system by only having a $+Q$-charged junction instead of a $+2 Q$-charged $\mathrm{DW}$ and a $-Q$-charged junction, effectively lowering the energy of the system by an amount equivalent to the magnetostatic energy associated with a DW. The other $\mathrm{P}$ configuration, where the TA is patterned on the narrow side of the V-shaped DW, does not offer the same rearrangement of the DW charge in the TA. The $+2 Q \mathrm{DW}$ experiences a barrier as it approaches the $+Q$ junction, and the DW energy is slightly higher when the DW is inside the trap than when it is outside, due to the concentration of the magnetic charge at the junction. A local potential well is nonetheless observed, as in order for the DW to leave the TA a transitory state where the charge of the junction is even higher than $+3 Q$ has to be created. The simulations show a pure filtering effect whereby only DWs in the $\mathrm{P}$ configuration are able to transmit through $\mathrm{T}$-shaped traps patterned on the wide side of the V-shaped DW, and DWs of the opposite chirality remain pressed against the trap for applied magnetic fields above the nucleation field of the structure (infinite barrier). The experiments suggest that the original DW does transmit in this case, although for a field on the order of the nucleation field of the structure, preserving the chirality filtering effect through the discrimination of the transmission field values. The qualitative agreement between experiments and simulations is otherwise excellent.

\section{ACKNOWLEDGMENT}

This work was supported by the European Community under the Sixth Framework Programme SPINSWITCH (Grant No. MRTN-CT-2006-035327).
${ }^{1}$ M. T. Bryan, T. Schrefl, and D. A. Allwood, Appl. Phys. Lett. 91, 142502 (2007).

${ }^{2}$ D. Petit, A.-V. Jausovec, D. Read, and R. P. Cowburn, J. Appl. Phys. 103, 114307 (2008).

${ }^{3}$ R. D. McMichael and M. J. Donahue, IEEE Trans. Magn. 33, 4167 (1997).

${ }^{4}$ D. A. Allwood, G. Xiong, M. D. Cooke, C. C. Faulkner, D. Atkinson, N. Vernier, and R. P. Cowburn, Science 296, 2003 (2002).

${ }^{5}$ D. A. Allwood, G. Xiong, M. D. Cooke, C. C. Faulkner, D. Atkinson, and R. P. Cowburn, J. Appl. Phys. 95, 8264 (2004).

${ }^{6}$ S. S. P. Parkin, M. Hayashi, and L. Thomas, Science 320, 190 (2008).

${ }^{7}$ D. A. Allwood, G. Xiong, C. C. Faulkner, D. Atkinson, D. Petit, and R. P. Cowburn, Science 309, 1688 (2005).

${ }^{8}$ C. A. Ross, F. J. Castaño, D. Morecroft, W. Jung, H. I. Smith, T. A. Moore, T. J. Hayward, J. A. C. Bland, T. J. Bromwich and A. K. Petford-Long, J. Appl. Phys. 99, 08S501 (2006).

${ }^{9}$ M. Kläui, C. A. F. Vaz, J. A. C. Bland, L. J. Heyderman, F. Nolting, A. Pavlovska, E. Bauer, S. Cherifi, S. Heun, and A. Locatelli, Appl. Phys. Lett. 85, 5637 (2004).

${ }^{10}$ M. Kläui, C. A. F. Vaz, J. Rothman, J. A. C. Bland, W. Wernsdorfer, G. Faini, and E. Cambril, Phys. Rev. Lett. 90, 097202 (2003).

${ }^{11}$ D. Backes, C. Schieback, M. Kläui, F. Junginger, H. Ehrke, P. Nielaba, U. Rüdiger, L. J. Heyderman, C. S. Chen, T. Kasama, R. E. Dunin-Borkowski, C. A. F. Vaz, and J. A. C. Bland, Appl. Phys. Lett. 91, 112502 (2007).

${ }^{12}$ M. Hayashi, L. Thomas, C. Rettner, R. Moriya, and S. S. P. Parkin, Nature (London) 3, 21 (2007)

${ }^{13}$ M. Kläui, C. A. F. Vaz, W. Wernsdorfer, E. Bauer, S. Cherifi, S. Heun, A. Locatelli, G. Faini, E. Cambril, L. J. Heyderman, and J. A. C. Bland, Physica B 343, 343 (2004).

${ }^{14}$ M. Kläui, H. Ehrke, U. Rüdiger, T. Kasama, R. E. DuninBorkowski, D. Backes, L. J. Heyderman, C. A. F. Vaz, J. A. C. Bland, G. Faini, E. Cambril, and W. Wernsdorfer, Appl. Phys.
Lett. 87, 102509 (2005).

${ }^{15}$ M. Hayashi, L. Thomas, C. Rettner, R. Moriya, X. Jiang, and S. S. P. Parkin, Phys. Rev. Lett. 97, 207205 (2006).

${ }^{16}$ J. Grollier, D. Lacour, V. Cros, A. Hamzic, A. Vaurès, A. Fert, D. Adam, and G. Faini, J. Appl. Phys. 92, 4825 (2002).

${ }^{17}$ C. K. Lim, T. Devolder, C. Chappert, J. Grollier, V. Cros, A. Vaurès, A. Fert, and G. Faini, Appl. Phys. Lett. 84, 2820 (2004).

${ }^{18}$ The OOMMF code is available at http://math.nist.gov/oommf.

${ }^{19}$ Y. Nakatani, A. Thiaville, and J. Miltat, J. Magn. Magn. Mater. 290-291, 750 (2005).

${ }^{20}$ M. Laufenberg, D. Backes, W. Bührer, D. Bedau, M. Kläui, U. Rüdiger, C. A. F. Vaz, J. A. C. Bland, L. J. Heyderman, F. Nolting, S. Cherifi, A. Locatelli, R. Belkhou, S. Heun, and E. Bauer, Appl. Phys. Lett. 88, 052507 (2006).

${ }^{21}$ D. Petit, A.-V. Jausovec, H. T. Zeng, E. Lewis, L. O’Brien, D. Read, and R. P. Cowburn, Appl. Phys. Lett. 93, 163108 (2008).

${ }^{22}$ A. Himeno, T. Okuno, T. Ono, K. Mibu, S. Nasu, and T. Shinjo, J. Magn. Magn. Mater. 286, 167 (2005).

${ }^{23}$ C. C. Faulkner, M. D. Cooke, D. A. Allwood, D. Petit, D. Atkinson, and R. P. Cowburn, J. Appl. Phys. 95, 6717 (2004).

${ }^{24}$ C. Chappert, H. Bernas, J. Ferré, V. Kottler, J.-P. Jamet, Y. Chen, E. Cambril, T. Devolder, F. Rousseaux, V. Mathet, and H. Launois, Science 280, 1919 (1998).

${ }^{25}$ S. O. Demokritov, C. Bayer, S. Poppe, M. Rickart, J. Fassbender, B. Hillebrands, D. I. Kholin, N. M. Kreines, and O. M. Liedke, Phys. Rev. Lett. 90, 097201 (2003).

${ }^{26}$ C. C. Faulkner, D. Atkinson, D. A. Allwood, and R. P. Cowburn, J. Magn. Magn. Mater. 319, 9 (2007).

${ }^{27}$ J. Fassbender and J. McCord, Appl. Phys. Lett. 88, 252501 (2006).

${ }^{28}$ G. Xiong, D. A. Allwood, M. D. Cooke, and R. P. Cowburn, Appl. Phys. Lett. 79, 3461 (2001).

${ }^{29}$ D. Petit, C. C. Faulkner, S. Johnstone, D. Wood, and R. P. Cowburn, Rev. Sci. Instrum. 76, 026105 (2005).

${ }^{30}$ O. Petracic, D. Read, and R. P. Cowburn, J. Appl. Phys. 101, 09F510 (2007) 